\title{
GAIT PARAMETER ADAPTATION TO ENVIRONMENTAL PERTURBATIONS IN QUADRUPEDAL ROBOTS
}

\author{
E. GARCIA*, J. ESTREMERA, P. GONZALEZ DE SANTOS and M. ARMADA \\ Industrial Automation Institute - CSIC, \\ 28500 La Poveda, Madrid, Spain \\ *E-mail: egarcia@iai.csic.es \\ www.iai.csic.es/users/egarcia
}

\begin{abstract}
Quadrupedal robots working outdoors are very slow robots prone to tumble down in the presence of perturbations. This paper presents a novel gaitadaptation method that enables walking-machine gaits to autonomously adapt to environmental perturbations, including the slope of the terrain, by finding the gait parameters that maximize robot's dynamic stability. Experiments with the SILO4 quadruped robot are presented and show how robot stability is more robust when the proposed approach is used for different external forces and sloping terrains.
\end{abstract}

Keywords: Quadruped robot; Gait adaptation; Dynamic stability margin.

\section{Introduction}

Walking robots designed for field and service applications usually perform statically stable gaits, which have been designed to optimize a static stability margin. Even when a dynamic stability margin is used to control the robot's motion, the gait pattern is a statically-stable one. As a result of this contradiction, the robot will still tumble down when confronted with any perturbing effect. Although an accurate dynamic stability margin measures robot stability, unstable situations are merely observed, not avoided, unless the gait pattern is modified. Existing dynamic gaits like the trot and the gallop require light, simplified robot designs with elastic actuators, which differ greatly from the heavy-limbed machines ${ }^{1,2}$ used for field and service applications. Therefore, statically stable robot gaits should be modified based on a more suitable dynamic stability margin to improve gait control.

Only a few researchers have attended to partially solve this problem, achieving adaptation to uneven terrain, yet they did not consider robot 


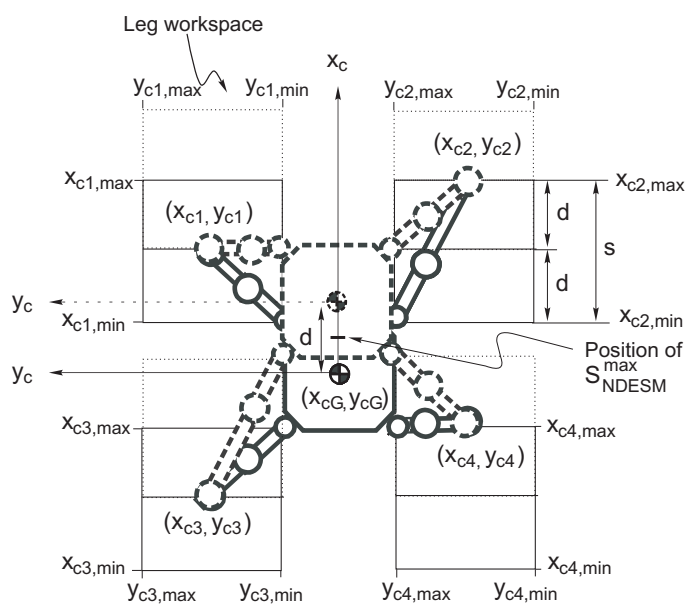

Fig. 1. Outlining of gait-parameter adaptation for a quadruped in the body-motion phase of a two-phase discontinuous gait

dynamics nor environmental perturbations. ${ }^{3,4}$ This paper presents a new method for gait adaptation to the environment based on the Normalized Dynamic Energy Stability Margin, $S_{N D E S M} .{ }^{5}$ The method maximizes the dynamic stability margin during the transfer and support phases and thus we get a more robust gait against external perturbations. Section 2 describes the gait-parameter adaptation approach. Experiments using the SILO4 walking robot (see Figure 5) are described in Section 3 to show the improvement of the gait-parameter adaptation method when external forces are applied.

\section{Gait Adaptation}

Let us assume a quadruped robot walking along the direction of its longitudinal body axis. Let us define an external, fixed reference frame $\{x, y, z\}$ and a body-fixed reference frame, $\left\{x_{c}, y_{c}, z_{c}\right\}$ centered at the robot's center of gravity $(\mathrm{CG})$ so that robot motion is along the $x_{c}$ axis and the $z_{c}$ axis is orthogonal to the body plane. The terrain can be inclined, forming an angle $\Psi$ with the $x$-axis (pitch angle) and an angle $\theta$ with the $y$-axis (roll angle). The gait optimization procedure is divided into the following two phases: 


\subsection{First phase: Leg-stroke and foothold calculation}

In this phase, foot and body CG positions in the body plane and the leg stroke that optimize the gait are determined. Figure 1 outlines a top view of leg workspaces, footholds and leg stroke for a quadruped during the bodymotion phase in a two-phase discontinuos gait. ${ }^{5}$ The four legs are in support, thus propelling the body forward. The initial position of the robot has been plotted in thick, solid line, while the final position of the robot has been plotted in thick, dashed line. Let us name $x_{c i}$ and $y_{c i}$ the $x_{c}$-coordinate and $y_{c}$-coordinate respectively of foothold $i$ referring to the body frame $\left\{x_{c}, y_{c}, z_{c}\right\}$ just before the body-motion phase starts. The CG trajectory has been also plotted $\left(x_{c G}, y_{c G}\right)$, finding the maximum-stability position at the middle of the trajectory, as explained before. The workspace of leg $i$ is delimited by $x_{c i_{\max }}, x_{c i_{\min }}, y_{c i_{\max }}, y_{c i_{\min }}$. Notice that the length of the body motion is half the leg stroke, that is, $s=2 d$. Therefore, maximizing $s$ is achieved by maximizing $d$, which can be expressed as a function of front legs' footholds:

$$
d\left(x_{c 1}, x_{c 2}\right)=x_{c 2}-x_{c 1} .
$$

The two rear legs' footholds can be expressed in terms of the two front legs' footholds:

$$
\begin{aligned}
& x_{c 3}=x_{c 3_{\text {min }}}+x_{c 2}-x_{c 1} \\
& x_{c 4}=x_{c 3_{\text {min }}}+2 x_{c 2}-2 x_{c 1} .
\end{aligned}
$$

The stroke pitch in the $y_{c}$-axis direction, $P_{y}$, provides the following relation to foothold $y_{c}$ components:

$$
\begin{aligned}
& y_{c 2}=y_{c 1}-P_{y} \\
& y_{c 4}=y_{c 3}-P_{y} .
\end{aligned}
$$

To obtain the optimized gait, $x_{c 1}, y_{c 1}, x_{c 2}, y_{c 3}, x_{c G}$ and $y_{c G}$ have to be obtained so that $S_{N D E S M}$ is maximum at the middle of the body-motion phase. This condition will equal the probabilities of losing robot stability when a rear leg is lifted or when a front leg is lifted, thus decreasing the overall probability of tumbling. Therefore, $S_{N D E S M}$ should be expressed as a function of footholds and CG position in the body reference frame, that is (see Ref. 6 for a more detailed explanation):

$$
S_{N D E S M}=\mathcal{F}\left(x_{c 1}, y_{c 1}, x_{c 2}, y_{c 3}, x_{c G}, y_{c G}, z_{c G}\right) \text {. }
$$

Taking into consideration that in this first phase of the optimization process the CG height is considered constant, $z_{c G}=h_{0} . S_{N D E S M}$ has 


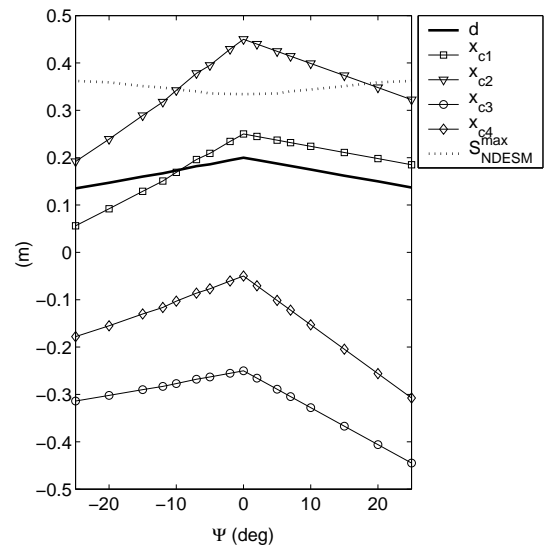

(a)

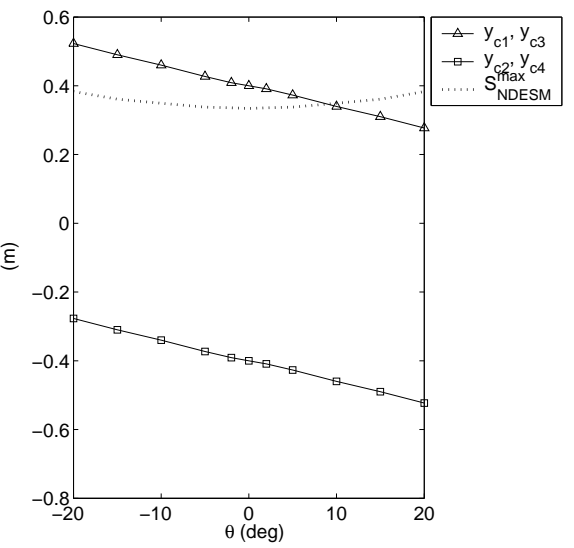

(b)

Fig. 2. Results of first-phase gait optimization for sloping terrain (a) Effect of pitch angle, (b) Effect of roll angle.

to be maximum when the body $\mathrm{CG}$ is located at the middle of the $\mathrm{CG}$ trajectory; therefore the objective function is:

$$
J_{1}=\mathcal{F}\left(x_{c 1}, y_{c 1}, x_{c 2}, y_{c 3}, x_{c G}+\frac{d}{2}, y_{c G}\right) .
$$

However, another objective function exists, because the leg stroke must be maximum too:

$$
J_{2}=d\left(x_{c 1}, x_{c 2}\right)
$$

with the following constraints:

$$
\begin{aligned}
& x_{c 1_{\min }} \leq x_{c 1} \leq x_{c 1_{\max }} \\
& x_{c 2_{\text {min }}} \leq x_{c 2} \leq x_{c 2_{\max }} \\
& y_{c 1_{\min }} \leq y_{c 1} \leq y_{c 1_{\max }} \\
& y_{c 3_{\min }} \leq y_{c 3} \leq y_{c 3_{\max }} \\
& 4 x_{c 1}-3 x_{c 2} \geq \xi_{1}-x_{c 1_{\max }}
\end{aligned}
$$

Constraints (9) to (12) are given by the leg-workspace limits, while constraint (13) avoids leg-workspace overlapping, where $\xi_{1} \geq 0$ is a constant.

To solve the multi-objective problem, the $\varepsilon$-Constraint Method is used. ${ }^{7}$ The problem has been solved numerically for the SILO4 robot in an iterative manner starting from an initial estimate of foot positions $x_{1}$ and $x_{2}$. Figures 2 and 3 show results of the first phase optimization for the medium-sized 


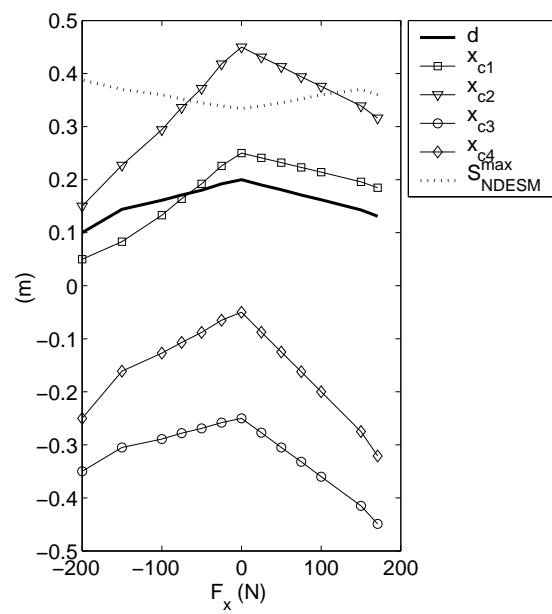

(a)

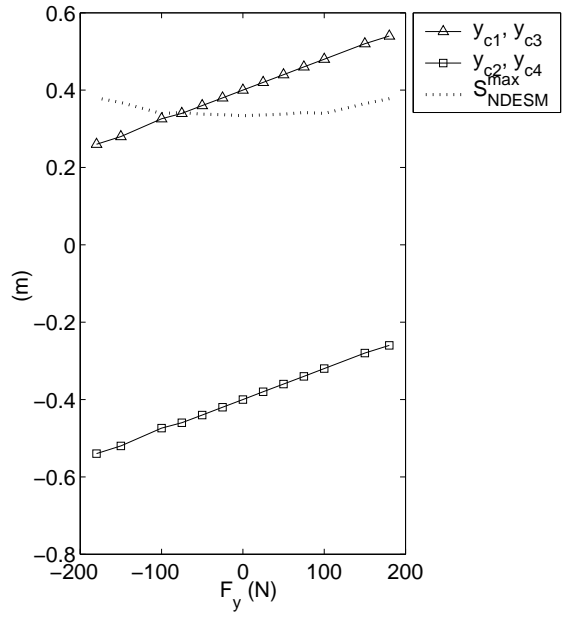

(b)

Fig. 3. Results of first-phase gait optimization for external forces (a) x component of force, (b) y component of force.

quadruped robot SILO4. Figure 2 shows footholds, leg stroke and maximum $S_{N D E S M}$ obtained for a robot walking on sloping terrain. As the terrain angle increases the robot has to modify foothold $x_{c}$ and $y_{c}$ components linearly to decrease the leg stroke (see d) and increase $S_{N D E S M}^{\max }$. Similar results have been found at the first phase optimization when external forces are applied (see Figures 3(a) and (b)). Note that the direct consequence of the linear reduction of leg stroke for increasing perturbations is a restriction in leg workspace. Thus, the bigger the disturbance, the bigger the reduction of leg workspace.

\subsection{Second phase: CG-height calculation}

When, as a result of the first phase of the optimization approach the leg stroke is reduced to adapt the walk to a stepped ground or an external force and enhance stability during the body-motion phase, stability is reduced during the transfer phase. This problem can be solved by reducing the CG height. Thus, the following condition is stated:

$$
\mathcal{F}(h)-S_{N D E S M}^{0}=0
$$

where $S_{N D E S M}^{0}$ stands for a constant value of the gait stability margin in nominal conditions, that is, over flat terrain when there are no external disturbances. The function $\mathcal{F}(h)$ is the result from the first phase of the 


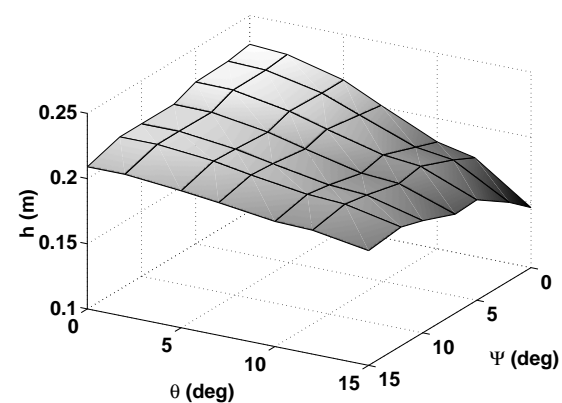

(a)

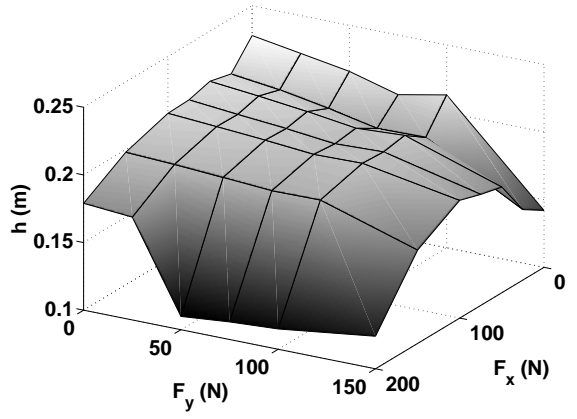

(b)

Fig. 4. Results of second-phase gait optimization for (a) sloping terrain, (b) external force.

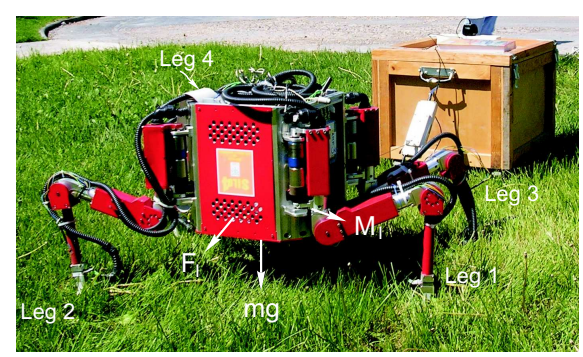

Fig. 5. Quadruped robot SILO4 pulling a 50-N load

optimization procedure, where every gait parameter has been obtained. Therefore, the only variable at this phase is CG height $(h)$. This problem is a nonlinear equation of a single variable that has been solved by leastsquares optimization.

Figure 4 shows results of CG height for the SILO4 walking (a) in sloping terrain and (b) when external forces are applied.

\section{Experiments with SILO4}

The improvement of gait stability obtained by the use of the gaitoptimization method herein proposed has been proved experimentally using the SILO4 robot $^{5,8}$ shown in Figure 5. A real service application has been simulated. The SILO4 robot walks while it pulls a load, thus being affected by an external force $F_{x}=-50 \mathrm{~N}$. As a consequence of the perturbation the robot finally tumbles down. Then, the experiment is repeated using the gait-optimization approach, which modifies the gait pattern for the $-50-\mathrm{N}$ 


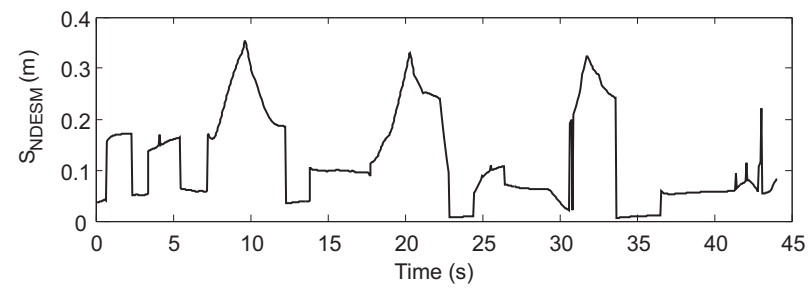

(a)

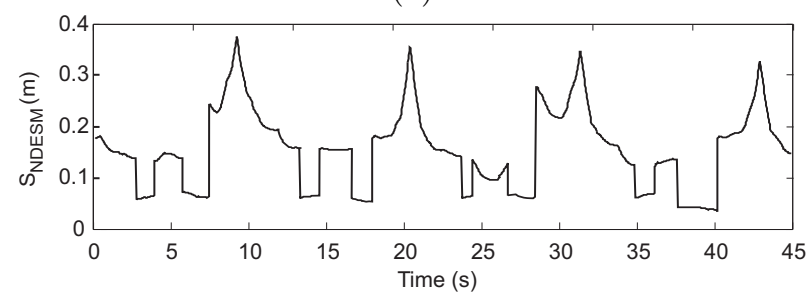

(b)

Fig. 6. Time evolution of $S_{N D E S M}$ for the SILO4 robot walking against a 50-N disturbance. (a) Standard gait, (b) Optimized gait using the proposed approach.

external force. In this experiment the robot pulls successfully the same load that made the robot tumble down in the previous experiment.

Figure 6(a) shows the evolution of the stability margin during the first experiment, when the SILO4 robot walks against the disturbance using a standard two-phase discontinuous gait. The stability margin drops during the transfer phase of the rear legs (because the destabilizing effects of leg transfer and force disturbance combine). Let us compare Figure 6(a) with Figure 6(b) which shows the evolution of the stability margin during the second experiment, when the SILO4 robot walks using the results of the gait optimization. As a result of adapting the gait to a $-50-\mathrm{N}$ force, the stability margin is symmetrical for the transfer phases of both the rear and the front legs. As a result of the adaptation to a $-50-\mathrm{N}$ force, the gait is more stable than in Figure 6(a), increasing the gait stability margin by $70 \%$ in this experiment. As shown in the gait optimization results, the use of the proposed gait-parameter adaptation approach increases the robot's robustness to external disturbances from a $50-\mathrm{N}$ load up to a $200-\mathrm{N}$ load, that is, four times the perturbation in this particular robot.

\section{Conclusions}

A new gait-adaptation method has been presented in this paper to cope with external perturbations. The method enables quadrupedal gaits to 
adapt to the slope of the terrain and external forces by finding the gait parameters that maximize robot stability. The resulting footholds of the gait optimization approach are linear functions of external forces and terraininclination angles. This reduces the computation time and enables the application of the gait-optimization method in real time.

Experimental results with the SILO4 robot have shown how robot stability is enhanced when the proposed approach is used to counteract external forces. The improvement in gait stability margin when applying the proposed gait-adaptation method to the SILO4 robot has been proved to be 70 percent for a $-50-\mathrm{N}$ external force. Also, the resulting gait increases the SILO4 robot's robustness to external disturbances from a $50-\mathrm{N}$ load up to a $200-\mathrm{N}$ load, that is, four times the perturbation in this particular robot. The proposed method has been shown to be of major relevance for the use of walking robots in field and service applications, where robots and environment interact.

\section{Acknowledgements}

This work has been funded by the Spanish Ministry of Education and Science through Grant DPI2004-05824.

\section{References}

1. P. Gonzalez de Santos, E. Garcia, J. Estremera and M. Armada, International Journal of Systems Science 36, 545 (2005).

2. T. Doi, R. Hodoshima, Y. Fukuda, S. Hirose, T. Okamoto and J. Mori, Journal of Robotics and Mechatronics 18, 318 (2006).

3. D. Wettergreen and C. Thorpe, Developing planning and reactive control for a hexapod robot, in Proc. IEEE Int. Conf. Robotics and Automation, (Atlanta, Georgia, 1996).

4. H. Tsukagoshi and S. Hirose, Intermittent crawl gait for quadruped walking vehicles on rough terrain, in Int. Conf. Climbing and Walking Robots, (Brussels, Belgium, 1998).

5. P. Gonzalez de Santos, E. Garcia and J. Estremera, Quadrupedal Locomotion: An Introduction to the Control of Four-Legged Robots (Springer-Verlag, London, 2006).

6. E. Garcia and P. Gonzalez de Santos, IEEE Transactions on Robotics 22, 1240 (2006).

7. V. Chankong and Y. V. Haimes, Multiobjective Decision MakingTheory and Methodology (Elsevier, New York, 1983).

8. Industrial Automation Institute, C.S.I.C., Madrid, Spain, The SILO4 Walking Robot, (2002). Available: http://www.iai.csic.es/users/silo4/. 